\title{
Contusiones en canales ovinas y su relación con el transporte ${ }^{\#}$
}

\author{
Bruising in lamb carcasses and its relationship with transport
}

\author{
JA Tarumán, CB Gallo* \\ Instituto de Ciencia Animal, Facultad de Ciencias Veterinarias, \\ Universidad Austral de Chile, Valdivia, Chile
}

\begin{abstract}
SUMMARY
The study was undertaken at one of the main slaughterplants of the Magallanes Region, in Chile. A total of 39,301 lamb and mutton carcasses were observed during the regular commercial slaughter, and the proportion of bruised carcasses was determined. The main characteristics of the vehicles and stocking densities used for the transportation of sheep was described; the sheep proceeded directly from farms located at distances up to $400 \mathrm{~km}$. Of all carcasses observed, $7.5 \%$ presented bruises; of the bruised carcasses $4.5 \%$ were classified as grade 1 (only subcutaneous tissue affected), $3 \%$ as grade 2 (subcutaneous and muscular tissues affected) and $0.005 \%$ as grade 3 (also bone affected). In terms of the approximate diameter of the lesions, $48 \%$ of them were classified as extension $1(<5 \mathrm{~cm}$ of diameter), $45 \%$ as extension 2 ( 6 to $10 \mathrm{~cm}$ of diameter) and only $7 \%$ as extension $3(>10 \mathrm{~cm}$ of diameter). The loin was the most commonly affected anatomical region in the carcass, concentrating $51.03 \%$ of the lesions. According to the transport distance, 7.6 and $8.1 \%$ of bruised carcasses were found in lambs that had been transported for $200-299 \mathrm{~km}$ and $300-400 \mathrm{~km}$, respectively; in contrast $6.1 \%$ and $6.7 \%$ of bruised carcasses were found in lambs transported for $0-99 \mathrm{~km}$ and $100-199 \mathrm{~km}$, also respectively. The stocking densities registered were in the range of 4.5 to $6.1 \mathrm{lambs} / \mathrm{m}^{2}$ (with a mean live weight of $28.5 \mathrm{~kg}$ ) and 3.8 to 5.3 mutton $/ \mathrm{m}^{2}$ (with a mean live weight of $37.9 \mathrm{~kg}$ ). It was concluded that the relatively low percentage of bruised carcasses found, with small lesions of reduced extension, is mainly attributable to the adequate structure of vehicles used for transport and the short distances travelled by the sheep.
\end{abstract}

Palabras clave: ovinos, transporte, canales, contusiones.

Key words: sheep, transport, carcasses, bruises.

\section{INTRODUCCION}

Prácticamente la totalidad de la carne de ovino que Chile exporta se origina en la Región de Magallanes (FIA 2000), en la cual se concentra aproximadamente el 57\% de la población total de 3.889.319 ovinos (INE 2007). Las exportaciones chilenas de carne ovina han experimentado una gran variabilidad en la última década, no sólo en cuanto a los volúmenes y montos comercializados, sino también en cuanto a los países de destino; en la actualidad los principales destinos son Canadá, Brasil, Israel, Japón, México, Rusia, países de la Unión Europea y Estados Unidos (SAG 2006 ${ }^{1}$ ). Cuando de exportaciones se trata, la calidad de la canal y de la carne adquieren mayor relevancia. Entre la amplia gama de factores que influyen en la calidad de la carne están aquellos relacionados con el trato de los animales durante su crianza, transporte y manejos inmediatamente previos al faenamiento (Warriss 1990, Grandin 1994). En Chile se ha visto que en bovinos los problemas más frecuentes debidos a estos factores son las pérdidas de peso, las contusiones en las canales y alteraciones de la calidad de la carne relacionadas

\footnotetext{
Aceptado: 05.03.2008.

\# Proyecto FONDECYT 1050492.

* Casilla 567, Valdivia, Chile; cgallo@uach.cl

1 SAG, Servicio Agrícola y Ganadero, Chile. 2006. Establecimientos Exportadores Pecuarios habilitados por Especie/Actividad. Disponible en http://www2.sag.gob.cl/Pecuaria/establecimientos_habilitados_exportar/habilitados_mercado/habilitados_mercado.htm
}

con el pH (Gallo 2004). Las contusiones afectan a todos los animales de abasto con particularidades que dependen de la especie y circunstancias antes de la muerte. Los ovinos, por su tamaño pequeño, ven fácilmente aprisionadas sus extremidades delgadas en grietas que se forman entre los suelos del embarque, las rampas elevadas y las paredes de los vehículos (Sacht 1994). Las fracturas que inutilizan al animal son raras; más comunes son las hemorragias subcutáneas o en el interior de los músculos, las que implican recortes y decomisos de trozos de la canal (Garriz 1995). Dado el potencial exportador de la Región de Magallanes y el hecho de que los mercados importadores son cada vez más exigentes, no sólo en términos de que la carne sea un alimento inocuo y saludable, sino que también tenga lo que se llama calidad ética, es decir, que los animales hayan sido manejados bajo sistemas que contemplen bienestar animal (Warriss 1990), se debe tener una mayor preocupación por el manejo en cada una de las etapas de la cadena de la carne ovina. Las canales con contusión no sólo generan pérdidas económicas cuantitativas por recortes a la industria, sino que además son el reflejo de malos manejos y pobre bienestar animal, reduciendo las posibilidades de alcanzar mercados más exigentes y de mayor precio. Considerando que en Chile sólo se han publicado estudios sobre las características y las consecuencias del transporte en bovinos (Gallo y col 2000, 2001, 2003, Gallo y Tadich 2004), el objetivo del presente trabajo fue describir algunas características del transporte de ovinos para faena en la Región de Magallanes, 
establecer la frecuencia de presentación de contusiones en las canales y determinar si existe relación con la distancia del transporte.

\section{MATERIAL Y METODOS}

El estudio se llevó a cabo durante febrero de 2005, en dependencias de una de las principales plantas faenadoras ovinas de exportación de la Región de Magallanes. Se registraron las características de los camiones cargados con ovinos que llegaron a la planta durante el período señalado y se analizó un total de 39.301 canales de corderos y borregos, las más frecuentes en la faena.

\section{CARACTERISTICAS DE LOS CAMIONES Y EL TRANSPORTE}

Se recolectaron antecedentes de los camiones que arribaban a la planta con el ganado, tales como tipo de camión (simple o con carro), material estructural (madera o metálico), número de pisos, número y clase de ovino transportado (corderos o borregos). La densidad de carga se determinó utilizando la información generada por el número de animales transportados por brete y las dimensiones de la superficie de carga de cada brete en los camiones y carros (ancho por largo utilizando una huincha métrica), y fue expresada en $\mathrm{kg}$ de peso vivo/ $\mathrm{m}^{2}$. Debido a que una vez desembarcados los animales del camión no eran pesados, se estimó el peso vivo utilizando el peso promedio de la canal de cada grupo de animales faenado según la planilla de faena de la planta y se asumió un rendimiento de la canal de $45 \%$ (Kusanovic 1978). Los ovinos eran clasificados por personal de la planta de acuerdo a sus características fenotípicas en corderos (machos y hembras menores de un año de edad aproximadamente) y borregos (machos y hembras entre 1 y 2 años de edad aproximadamente).

Para determinar la distancia del transporte se revisaron los registros del frigorífico y se identificó la estancia de procedencia para cada lote, para luego calcular las distancias recorridas según un mapa rutero, clasificándolas arbitrariamente en cuatro intervalos: hasta $99 \mathrm{~km}, 100-199 \mathrm{~km}$, 200-299 km y 300-400 km. Se determinó el porcentaje de canales con contusión para cada tramo.

\section{OBSERVACION DE LAS CONTUSIONES}

Se realizó la inspección visual de las canales faenadas diariamente durante el período en estudio, registrando en ellas la presencia o ausencia de contusiones y las características de éstas, tales como el grado, la ubicación y la extensión.

Grado de contusión: en el caso de las canales que presentaron algún tipo de lesión, se anotó el grado de la contusión de acuerdo al daño o destrucción de los tejidos, según se encuentra definido en la Norma Chilena de Tipificación de Canales Bovinas (INN 2002), siendo las de grado 1 aquellas que afectan el tejido subcutáneo solamente, las de grado 2 las que alcanzan los tejidos subcutáneo y muscular y las de grado 3 las que comprometen los tejidos anteriores más el tejido óseo.

Extensión de la contusión: cada contusión fue evaluada según el diámetro aproximado del área afectada y se consideraron tres niveles: extensión $1=$ hasta $5 \mathrm{~cm}$ de diámetro, extensión $2=6$ a $10 \mathrm{~cm}$ de diámetro y extensión 3 = mayor a $10 \mathrm{~cm}$ de diámetro.

En las canales en que se presentó más de una contusión de diferente grado y extensión primó el criterio de la contusión de mayor magnitud para calificarla.

Ubicación anatómica de la contusión: se individualizaron 4 regiones anatómicas que fueron pierna, paleta, lomo y tórax-abdomen (figura 1).

Para el análisis de los resultados se utilizó estadística descriptiva mostrando frecuencias y porcentajes. Para determinar asociación entre distancia de transporte y presencia de contusiones se analizó el caso de los corderos solamente (más frecuentes y procedentes de todos los tramos) y se utilizó la prueba estadística de "Ji-cuadrado" comparando la frecuencia de contusiones en las canales de los corderos provenientes de entre 1 y $200 \mathrm{~km}$ y aquellos procedentes de entre 201 y $400 \mathrm{~km}$.

\section{RESULTADOS Y DISCUSION}

Durante el período en estudio se analizaron un total de 69 cargas que arribaron con 39.301 ovinos, de los cuales 34.815 eran corderos (peso vivo promedio estimado de 28,5 $\mathrm{kg}$ ) y 4.486 eran borregos (peso vivo promedio estimado de $37,9 \mathrm{~kg}$ ). Esto corresponde a una muestra de aproximadamente el $7 \%$ de los ovinos que se faenan anualmente en todas las plantas faenadoras de carne de la Región de Magallanes (SAG 2005²). Los 7 camiones que arribaron a la planta faenadora en forma repetida transportando los ovinos durante el período del estudio eran todos con carro, cinco de ellos eran completamente de metal y dos tenían partes de metal y madera (piso y bordes de madera). Todos eran de tres pisos, cada piso estaba dividido en 8 a 10 bretes; dependiendo de la clase de ovino, se transportaban 12 a 14 corderos o 10 a 12 borregos por brete. El rango de densidad de carga estimado fue de 106,8-225,4 $\mathrm{kg} / \mathrm{m}^{2}$ para los borregos y de $112,1-216,4 \mathrm{~kg} / \mathrm{m}^{2}$ para los corderos. Estos rangos de densidad de carga son similares a los encontrados por Warriss y col (2003), quienes registraron $121-214 \mathrm{~kg} / \mathrm{m}^{2}$ en transportes comerciales en el Reino Unido. En cuanto a la disponibilidad de espacio por animal ( $\mathrm{m}^{2} /$ ovino), dichas densidades implican que se

SAG, Servicio Agrícola y Ganadero, Chile. 2005. Faenamiento Regional Temporada 1987-2004. Disponible en: http://magallanes. sag.gob.cl/faenam.PDF 
otorgan 0,16 a $0,22 \mathrm{~m}^{2}$ /ovino en el caso del transporte de corderos y 0,19-0,26 $\mathrm{m}^{2}$ /ovino, en el caso de borregos. En este sentido, la Unión Europea (2005) establece una superficie para transporte en carretera de 0,2 a $0,3 \mathrm{~m}^{2}$ por ovino esquilado de más de $26 \mathrm{~kg}$ y menos de $55 \mathrm{~kg}$. Para ovinos no esquilados, menores de $55 \mathrm{~kg}$, se debe aumentar el espacio disponible entre 0,3 a $0,4 \mathrm{~m}^{2}$ y para corderos pequeños, menores a $26 \mathrm{~kg}$, puede disponerse de una superficie inferior a $0,2 \mathrm{~m}^{2}$. En el Reino Unido el Farm Animal Welfare Council (FAWC 1991) recomienda una disponibilidad de espacio de aproximadamente $0,2 \mathrm{~m}^{2} /$ cordero de $30 \mathrm{~kg}$ y en Nueva Zelanda el Animal Welfare Advisory Committee (AWAC 1996) recomienda 0,17 y $0,21 \mathrm{~m}^{2} /$ cordero para individuos de 30 y $40 \mathrm{~kg}$ respectivamente. Otros estudios, como el realizado por Cockram y col (1996), establecen un rango entre $0,22-0,41 \mathrm{~m}^{2} /$ ovino. Lo más frecuente en Chile es que los corderos no se esquilen antes de ser enviados a matadero, por lo cual el espacio disponible está en el extremo inferior del rango recomendado por otros autores. Densidades muy altas durante el transporte son perjudiciales para el bienestar de los ovinos (Warriss y col 2003); además impiden que los animales se echen, lo que puede causar fatiga y daño muscular, considerando que éstos tienden a echarse luego de aproximadamente $4 \mathrm{~h}$ de viaje (Knowles y col 1998). El hecho de que se tienda en general a usar altas densidades de carga en el transporte animal (Gallo y col 2005) se explica por razones económicas, ya que al cargar el máximo número de animales posible en los camiones se disminuyen los costos del transporte (Warriss y col 2001). En Chile ayuda a esto la inexistencia de una reglamentación de transporte para ovinos.

El porcentaje de canales con contusiones fue en general de 7,5\%; en el análisis por clase se presentó un 7,4\% de contusiones en corderos y un $8,9 \%$ en los borregos. Respecto al grado de las contusiones, se registró en general un 4,5\% de contusiones grado 1 , un $3 \%$ grado 2 y un 0,005 grado 3 . La frecuencia de presentación de contusiones encontrada es menor a la registrada por Carter (2007) en ovinos de la Región de Aysén, quien encontró un $25 \%$ de contusiones en corderos faenados localmente tras $12 \mathrm{~h}$ de transporte $\mathrm{y}$ un $33 \%$ en aquellos con transporte prolongado (46 h) hasta la Región de Los Lagos. Cockram y Lee (1991), en un estudio realizado en Edinburgo, registraron un 69\% de canales de cordero con contusiones, de las cuales un $12 \%$ se clasificó como severamente contusas, en animales provenientes directamente del predio; dicho porcentaje aumentó en un $20 \%$ en los animales que pasaron previamente por un mercado de ganado. En otro estudio realizado en el Reino Unido, Jarvis y Cockram (1995) estimaron que el $26 \%$ de los ovinos tenía algún grado de contusión. La baja frecuencia de canales con contusión encontrada en el presente estudio se podría relacionar con el corto trayecto recorrido, la buena estructura de los camiones, la inexistencia de ferias ganaderas que actúen como intermediarios entre las estancias y las plantas faenadoras, así

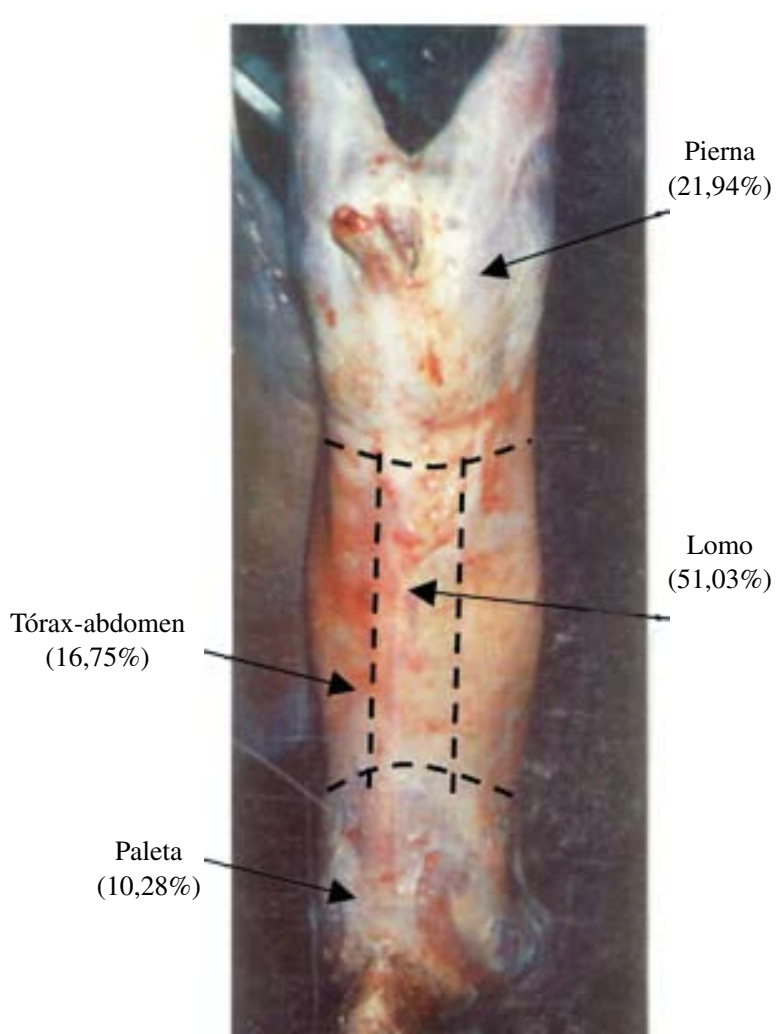

Figura 1. Regiones anatómicas consideradas para la ubicación de las contusiones en las canales ovinas y porcentaje de contusiones encontrado en cada una de ellas.

Anatomical regions considered for the location of the bruises in sheep carcasses and percentage of bruises observed in each of them.

Cuadro 1. Distribución (\%) de las canales de corderos y borregos con contusión según la extensión (diámetro aproximado) de las lesiones.

Distribution (\%) of the lamb and mutton carcasses with bruising, according to the extension (approximate diameter) of the lesions.

\begin{tabular}{lccc}
\hline \multirow{2}{*}{ Clase de ovino } & \multicolumn{3}{c}{ Extensión de las contusiones } \\
\cline { 2 - 4 } & $1(1-5 \mathrm{~cm})$ & $2(6-10 \mathrm{~cm})$ & $3(>10 \mathrm{~cm})$ \\
\hline Corderos & 47,2 & 45,5 & 7,3 \\
Borregos & 52,9 & 40,4 & 6,7 \\
\hline Total & 48,0 & 44,8 & 7,2 \\
\hline
\end{tabular}

como a la difusión que ha hecho la planta estudiada sobre la importancia del buen manejo de los corderos.

En las dos clases de ovinos predominaron las contusiones que abarcaron superficies de pequeña extensión $(<5 \mathrm{~cm})$; las contusiones $>10 \mathrm{~cm}$ fueron las menos frecuentes (cuadro 1). Los resultados son concordantes con los de Jarvis y Cockram (1995) y Jarvis y col (1996), quienes encontraron que la mayoría de las contusiones en ovinos tenía una extensión menor a $5 \mathrm{~cm}$. En Chile, Carter (2007) también encontró lesiones similares en cuanto a extensión, señalando que el 100\% de las contusiones medía 
menos de $5 \mathrm{~cm}$ en los corderos faenados localmente en Puerto Aysén (con 12 horas de transporte), en tanto con el transporte prolongado hacia la zona centro sur (46 horas de transporte) se incrementó la extensión y gravedad de las lesiones, registrándose un $52 \%$ de contusiones de extensión menor a $5 \mathrm{~cm}$, un $38 \%$ entre 6 y $10 \mathrm{~cm}$ y un $10 \%$ mayor a $10 \mathrm{~cm}$; además, tras el transporte prolongado este autor encontró un $3 \%$ de contusiones grado 2 , inexistentes tras el transporte de $12 \mathrm{~h}$. La presencia de estas lesiones de pequeña extensión podría atribuirse no sólo al transporte, sino también a la carga y descarga, cuando los corderos se toman del vellón o de diferentes partes del cuerpo cuando se niegan a avanzar, o al subirlos y bajarlos manualmente de un camión, manejos que fueron frecuentemente observados. El lomo fue la región anatómica más afectada por contusiones, concentrando un $51,03 \%$ de las lesiones (figura 1), valores que concuerdan con un estudio realizado por Cockram y Lee (1991). Por otra parte, la región anatómica que se vio menos afectada fue la paleta, en tanto pierna y tórax-abdomen registraron un $21,94 \%$ y $16,75 \%$ respectivamente (figura 1). El lomo es una de las regiones más valiosas de la canal y cuando se presentan contusiones grado 2 , se deben realizar recortes que pueden abarcar desde pequeñas zonas en que sólo se pierden unos gramos, hasta varios kilos de carne por animal. Canales con demasiados recortes, ya sea a causa de contusiones u otros problemas, deben ser descartadas de exportación e incluso puede ocurrir el decomiso de la canal completa y las consiguientes pérdidas económicas. De acuerdo a los resultados de este estudio, al menos en este frigorífico, las pérdidas por contusión no serían muy importantes, aunque en futuros estudios sería interesante poder determinar el peso de los recortes. Por otra parte, todo indica que el transporte es un proceso que no sólo implica el viaje propiamente tal, sino manejos adicionales que van desde el arreo de los animales en el predio de origen, carga de los animales al camión, descarga, además del manejo en la planta faenadora, y que debería estudiarse como cadena completa, además de responsabilizar a todos los eslabones comprometidos (productores, transportistas, intermediarios cuando los hay y plantas faenadoras), tal como lo señala la OIE (2005) en sus directrices sobre bienestar animal.

De acuerdo al origen de las cargas (camionadas), se observó que eran provenientes de sectores regionales con un radio de hasta $400 \mathrm{~km}$ de distancia. En el presente trabajo se pudo apreciar que a medida que aumentaba el tramo recorrido por los animales, aumentaba el porcentaje de contusiones tanto en los corderos como en los borregos, aunque estos últimos provenían sólo de los dos tramos más alejados de la planta faenadora (cuadro 2). Un análisis estadístico de la presencia de contusiones demostró que los corderos de los dos tramos de recorrido más cortos tenían menos contusiones que aquellos de los dos tramos mayores $(\mathrm{P}<0,01)$, concluyéndose que hubo asociación entre la distancia recorrida y la presencia de contusiones. Cabe destacar que la relación entre distancia recorrida y contusiones no es siempre directa, probablemente porque hay muchos factores que interactúan. Knowles y col (1994) encontraron que en corderos la distancia recorrida fue un pobre predictor de presentación de contusiones. Hartung (2003) incluso sostiene que las contusiones y heridas suceden más a menudo en transportes de corta distancia. Sin embargo, hay trabajos en ovinos en los que se señala que sí existe relación entre distancia de transporte y contusiones, como los efectuados por Cockram y Lee (1991), Jarvis y col (1996), Knowles (1998). En el caso de la Región de Magallanes es importante señalar que algunas rutas, aunque cortas, incluyen tramos de ripio o con muchas curvas y otras incluyen cruces cortos en barcazas, lo que puede influir sobre la presencia de contusiones, además de aumentar el tiempo de transporte, sobre todo cuando se debe esperar para el cruce en barcaza.

Del presente estudio se concluye que el tipo de camión utilizado en todas las cargas registradas en esta planta faenadora de la Región de Magallanes está especialmente diseñado para la especie ovina y que la densidad de carga utilizada, aun dentro de los rangos registrados por la

Cuadro 2. Frecuencia (número y \%) de presentación de canales de corderos y borregos con contusión en relación con la distancia del transporte y la profundidad (grado) de la lesión. (grade) of the lesion.

Frequency (number and \%) of presentation of bruised lamb and mutton carcasses in relation to the distance of the transport and the depth

\begin{tabular}{|c|c|c|c|c|c|c|c|c|}
\hline \multirow{2}{*}{$\begin{array}{l}\text { Distancia } \\
\text { recorrida }\end{array}$} & \multirow{2}{*}{ Corderos } & \multirow{2}{*}{$\begin{array}{c}\% \\
\text { canales contusas }\end{array}$} & \multicolumn{6}{|c|}{ Canales contusas } \\
\hline & & & Grado 1 & $\%$ & Grado 2 & $\%$ & Grado 3 & $\%$ \\
\hline 000-99 km & 5.095 & 6,1 & 202 & 66,0 & 107 & 34,0 & 0 & 0 \\
\hline $100-199 \mathrm{~km}$ & 6.665 & 6,7 & 270 & 61,1 & 174 & 38,8 & 1 & 0,1 \\
\hline $200-299 \mathrm{~km}$ & 10.624 & 7,6 & 456 & 56,5 & 350 & 43,4 & 1 & 0,1 \\
\hline \multirow[t]{2}{*}{$300-400 \mathrm{~km}$} & 12.431 & 8,1 & 614 & 60,5 & 393 & 39,5 & 0 & 0 \\
\hline & Borregos & & & & & & & \\
\hline $200-299 \mathrm{~km}$ & 3.285 & 7,0 & 172 & 74,1 & 60 & 25,9 & 0 & 0 \\
\hline $300-400 \mathrm{~km}$ & 1.201 & 9,0 & 60 & 55,6 & 48 & 44,4 & 0 & 0 \\
\hline
\end{tabular}


literatura internacional, se acerca más al límite superior. Respecto a las contusiones, el porcentaje registrado fue relativamente bajo y aumentó a medida que aumentaba la distancia recorrida.

\section{RESUMEN}

El estudio se llevó a cabo en una de las principales plantas faenadoras de carnes en la Región de Magallanes. De un total de 39.301 canales de corderos y borregos observadas dentro de la faena habitual, se determinó el porcentaje de canales que presentó contusiones. Además se describieron algunas características de los camiones que transportan ovinos y se determinó la densidad de carga general utilizada. Los ovinos procedían directamente de estancias regionales ubicadas hasta $400 \mathrm{~km}$ de distancia. Se registró un 7,5\% de contusiones en el total de las canales, de las cuales $4,5 \%$ correspondió a contusiones de grado 1 (afectan sólo tejido subcutáneo), un 3\% de grado 2 (afectan también músculo) y $0,005 \%$ de grado 3 (afectan incluso hueso). En cuanto a la extensión de las lesiones, $48 \%$ fue $<5 \mathrm{~cm}$ de diámetro aproximado, $44,8 \%$ de 6 a $10 \mathrm{~cm}$ y sólo $7,2 \%>10 \mathrm{~cm}$. En relación a la ubicación anatómica de las lesiones, el lomo fue la región más afectada, concentrando un 51,03\% de las lesiones. Respecto a la frecuencia de presentación de contusiones en relación a la distancia del transporte, para el caso de los corderos se encontró un 7,6 y 8,1\% de canales contusas en los dos tramos más largos (200-299 km y 300-400 km), en cambio, en los dos tramos más cortos hubo un 6,1\% (0-99 km) y un 6,7\% (100-199 km). La densidad de carga registrada estuvo en el rango de 4,5 a 6,1 corderos $/ \mathrm{m}^{2}$ (con un peso vivo promedio de $28,5 \mathrm{~kg}$ ) y 3,8 a 5,3 borregos $/ \mathrm{m}^{2}$ (peso vivo promedio de $37,9 \mathrm{~kg}$ ). Se concluye que la frecuencia de canales con contusión encontrada en los corderos y borregos en el presente estudio es relativamente baja, con lesiones de poca profundidad y extensión, lo que se atribuye principalmente a una adecuada estructura de los camiones y al corto trayecto recorrido por los animales.

\section{AGRADECIMIENTOS}

Los autores agradecen al Frigorífico Simunovic S.A. y al Servicio Agrícola y Ganadero las facilidades otorgadas para realizar este estudio.

\section{REFERENCIAS}

AWAC, Animal Welfare Advisory Committee. 1996. Amendments to the Code of Recommendations and Minimum Standards for the welfare of animals transported within New Zealand. New Zealand Government Ministry of Agriculture and Fisheries, Wellington, New Zealand.

Carter LM. 2007. Efectos del transporte prolongado terrestre marítimo sobre pérdidas de peso vivo y características de la canal en corderos. Memoria de título, Escuela de Medicina Veterinaria, Universidad Austral de Chile, Valdivia, Chile.

Cockram M, R Lee. 1991. Some preslaughter factors affecting the occurrence of bruising in sheep. Br Vet J 147, 120-125.

Cockram M, J Kent, E Goddard, N Waran, I McGilp, R Jackson, G Muwanga, S Prytherch. 1996. Effect of space allowance during transport on the behavioural and physiological responses of lambs during and after transport. J Anim Sci 62, 461-467.

FAWC, Farm Animal Welfare Council. 1991. Report on the European Commission proposals on the transport of animals. MAFF Publications, London, UK.

FIA, Fundación para la Innovación Agraria, Chile. 2000. Ministerio de Agricultura. Estrategia de innovación agraria para producción de carne ovina.
Gallo C, S Pérez, C Sanhueza, J Gasic. 2000. Efectos del tiempo de transporte de novillos previo al faenamiento sobre el comportamiento, pérdidas de peso y algunas características de la canal. Arch Med Vet 32, 157-170.

Gallo C, M Espinoza, J Gasic. 2001. Efectos del transporte por camión durante 36 horas con y sin período de descanso sobre el peso vivo y algunos aspectos de calidad de carne en bovinos. Arch Med Vet 33, 43-53.

Gallo C, G Lizondo, T Knowles. 2003. The effects of journey and lairage time on steers transported to slaughter in Chile. Vet Rec 152, 361-364.

Gallo C. 2004. Bienestar animal y calidad de carne durante los manejos previos al faenamiento en bovinos. XXXII Jornadas Uruguayas de Buiatría, Montevideo, Uruguay, Pp 147-157.

Gallo C, N Tadich. 2004. Bienestar animal y calidad de carne durante los manejos previos al faenamiento en bovinos. Parte 1. Vetermas 5, 2-6.

Gallo C, P Warriss, T Knowles, R Negrón, A Valdés, I Mencarini. 2005. Densidades de carga utilizadas para el transporte de bovinos destinados a matadero en Chile. Arch Med Vet 37, 155-159.

Garriz C. 1995. Calidad total vs. "rechazos y machucones". INTA Argentina CCDH, 10-12.

Grandin T. 1994. Farm animal welfare during handling, transport and slaughter. Anim Welfare Forum 204, 372-377.

Hartung J. 2003. Effects of transport on health of farm animals. Vet Res Comm 1, 525-527.

INE, Instituto Nacional de Estadísticas, Chile. 2007. VII Censo Nacional Agropecuario. Total Nacional.

INN, Instituto Nacional de Normalización, Chile. 2002. Norma Chilena de Tipificación de Canales Bovinas. NCH.1306.Of. 2002.

Jarvis A, M Cockram. 1994. Effects of handling and transport on bruising of sheep sent directly from farms to slaughter. Vet Rec 135, 523-525.

Jarvis A, M Cockram. 1995. Handling of sheep at markets and the incidence of bruising. Vet Rec 136, 582-585.

Jarvis A, M Cockram, I McGilp. 1996. Bruising and biochemical measures of stress, dehydration and injury determined at slaughter in sheep transported from farms or markets. Br Vet J 152, 719-722.

Knowles T, D Maunder, P Warris. 1994. Factors affecting the incidence of bruising in lambs arriving at one slaughterhouse. Vet Rec 134, 107-110.

Knowles T. 1998. A review of the road transport of slaughter sheep. Vet Rec 143, 212-219.

Knowles T, P Warriss, S Brown, J Edwards. 1998. Effects of stocking density on lambs being transported by road. Vet Rec 142, 503-509.

Kusanovic S. 1978. Consideraciones del peso de la res en la producción de carne ovina. Instituto Tecnológico de Carnes, Universidad Austral de Chile. Informativo sobre carne y productos cárneos $3,16-24$.

OIE, World Organization for Animal Health. 2005. Terrestrial animal health code 2005. Part 3. Section 3.7. Animal welfare. Chapter 3.7.3. Guidelines for the transport of animals by land.

Sacht J. 1994. Amtstierärztliche Untersuchung der Tiere sowie Feststellung der Voraussetzungen für den Tieren auf dem Transport. DVG - Tagung, "Hygiene und Tierschutz beim Tiertransport", Pp 8-9.

Unión Europea. 2005. Reglamento (CE) No 1/2005 del Consejo de 22 de diciembre de 2004.

Warriss PD. 1990. The handling of cattle preslaughter and its effects on carcass and meat quality. Appl Anim Behav Sci 28, 171-186.

Warriss P, S Edwards, S Brown, T Knowles. 2001. A survey of the stocking densities at which sheep are transported commercially in the United Kingdom. Vet Rec 149, 1-6.

Warriss P, S Brown, T Knowles. 2003. Assessment of possible methods for estimating the stocking density of sheep being carried on commercial vehicles. Vet Rec 153, 315-319. 
